\title{
A Note on Elliptic Curves Over Finite Fields
}

\author{
By Hans-Georg Rück
}

\begin{abstract}
Let $E$ be an elliptic curve over a finite field $k$ and let $E(k)$ be the group of $k$-rational points on $E$. We evaluate all the possible groups $E(k)$ where $E$ runs through all the elliptic curves over a given fixed finite field $k$.
\end{abstract}

Let $k$ be a finite field with $q=p^{n}$ elements. An elliptic curve $E$ over $k$ is a projective nonsingular curve given by an equation

$$
Y^{2} Z+a_{1} X Y Z+a_{3} Y Z^{2}=X^{3}+a_{2} X^{2} Z+a_{4} X Z^{2}+a_{6} Z^{3}
$$

with coefficients $a_{1}, \ldots, a_{6}$ in $k$. For each field $\tilde{k}$ that contains $k$, the set $E(\tilde{k})$ of points with coordinates in $\tilde{k}$ satisfying (1) forms an Abelian group whose zero element can be chosen as the element $(0,1,0)$. In this note we want to look at the following Question 1: Given a fixed finite field $k$, what are the possible Abelian groups $E(k)$, when the coefficients of the equation (1) vary over all the possible values in $k$ ? The answer to this question is given in Theorem 3. If we just look at the possible orders \#E(k), the appropriate Question 2 was answered by Waterhouse [4] (see also Deuring [1] for $k=\mathbf{F}_{p}$ ) using the theorem of Honda and Tate [3] for Abelian varieties over finite fields.

TheOREM 1a [4]. All the possible orders ${ }^{*} h=\# E(k)$ are given by $h=1+q-\beta$, where $\beta$ is an integer with $|\beta| \leqslant 2 \sqrt{q}$ satisfying one of the following conditions:

(a) $(\beta, p)=1$;

(b) If $n$ is even: $\beta= \pm 2 \sqrt{q}$;

(c) If $n$ is even and $p \not \equiv 1 \bmod 3: \beta= \pm \sqrt{q}$;

(d) If $n$ is odd and $p=2$ or $3: \beta= \pm p^{(n+1) / 2}$;

(e) If either (i) $n$ is odd or (ii) $n$ is even, and $p \not \equiv 1 \bmod 4: \beta=0$.

Following the general ideas of Waterhouse [4] we can also give an answer to the first question.

For an elliptic curve $E$ over $k$ let $\operatorname{End}(E)$ be the ring of group endomorphisms of $E$ which are given by algebraic equations with coefficients in $k$. It is known that $\operatorname{End}(E)$ is an order in a finite-dimensional division algebra over $\mathbf{Q}$. This division algebra determines \#E(k):

THEOREM 2 [2]. Let $E, E^{\prime}$ be elliptic curves over $k$; then

$$
\# E(k)=\# E^{\prime}(k) \quad \text { if and only if } \operatorname{End}(E) \otimes_{\mathbf{Z}} \mathbf{Q}=\operatorname{End}\left(E^{\prime}\right) \otimes_{\mathbf{Z}} \mathbf{Q} \text {. }
$$

Received July 9, 1986.

1980 Mathematics Subject Classification (1985 Revision). Primary 11G20.

*Here "possible orders" or "possible groups" mean that these orders or groups really occur. 
There is a special endomorphism $\pi$, called the Frobenius endomorphism, which maps a point $P=(x, y, z)$ on $E$ to $\pi(P)=\left(x^{q}, y^{q}, z^{q}\right)$ on $E$. From this definition it follows immediately that $E(k)$ is the set of all the points $P$ on $E$ with $\pi(P)=P$.

If $h$ is a fixed possible order \#E(k), then by Theorem 2 the division algebra $K=\operatorname{End}(E) \otimes_{\mathbf{Z}} \mathbf{Q}$ is fixed. What are the orders in $K$ that are rings of endomorphisms of elliptic curves over $k$ ? The answer is:

THEOREM 1b [4]. Let $h=1+q-\beta$ be a possible order $\# E(k)$, where $\beta$ satisfies one of the conditions (a),.., (e) of Theorem $1 \mathrm{a}$.

In case $(\mathrm{a}): K=\mathbf{Q}(\pi)$ is an imaginary quadratic field over $\mathbf{Q}$; all the orders in $K$ are possible endomorphism rings.

In case (b): $K$ is a division algebra of order 4 with center $\mathbf{Q}, \pi$ is a rational integer, all the maximal orders in $K$ are possible endomorphism rings.

In cases (c), (d), (e): $K=\mathbf{Q}(\pi)$ is an imaginary quadratic field over $\mathbf{Q}$, all the orders in $K$ whose conductor is prime to $p$ are possible endomorphism rings.

Let $h$ be a possible order and $h=\prod_{l} l^{h_{l}}$ its decomposition in powers of prime numbers. Since the genus of an elliptic function field is one, the possible $E(k)$ with $\# E(k)=h$ are among all the groups of the form

$$
\mathbf{Z} / p^{h_{r}} \mathbf{Z} \times \prod_{l \neq p}\left(\mathbf{Z} / l^{a_{l}} \mathbf{Z} \times \mathbf{Z} / l^{h_{l}-a_{l}} \mathbf{Z}\right) \quad \text { with } 0 \leqslant a_{l} \leqslant h_{l} .
$$

The relation between $\operatorname{End}(E)$ and the structure of $E(k)$ is given by the following lemma:

Lemma 1. Let $m$ be a positive integer which is not divisible by $p$, and let $E_{m}$ be the group of the points $P$ on $E$ with $m P=0$. Then $E_{m}$ is contained in $E(k)$ if and only if $\pi-1$ is divisible by $m$ in $\operatorname{End}(E)$.

Proof. If $\pi-1$ is divisible by $m$ in $\operatorname{End}(E)$, then $\pi-1=\lambda \cdot m$ with $\lambda \in \operatorname{End}(E)$. Let $P \in E_{m}$, then $(\pi-1)(P)=\lambda \cdot m(P)=0$. Hence $\pi(P)=P$ and $E_{m} \subset E(k)$.

If $E_{m} \subset E(k)$, then the kernel of $\pi-1$ contains the kernel of the multiplication by $m$. Since the multiplication by $m$ is separable, the universal mapping property for Abelian varieties (see [5, p. 27, Proposition 10]) shows that $\pi-1=m \cdot \lambda$ with $\lambda \in \operatorname{End}(E)$.

Lemma 2. We assume that $\pi$ is not contained in $\mathbf{Q}$; then by Theorem $1 \mathrm{~b}$ the division algebra $K$ is an imaginary quadratic field. The maximal order in $K$ is denoted by $O_{K}$. Let $l$ be a rational prime number which is different from $p$ and suppose that $\pi-1=l^{x} \cdot \omega$, where $\omega \in O_{K}$ is not divisible by $l$. Then

$$
x=\min \left\{v_{l}(q-1),\left[\frac{v_{l}(\# E(k))}{2}\right]\right\} .
$$

$\left([\lambda]\right.$ is the largest rational integer $\leqslant \lambda ; v_{l}(\cdot)$ is the normalized valuation of $\mathbf{Z}$ corresponding to $l$.)

Proof. The zeta function of $E$ yields the equation

$$
\# E(k)=(\pi-1)(\bar{\pi}-1)=q-(\pi+\bar{\pi})+1 .
$$


From this we get the two equations

$$
\# E(k)=l^{2 x} \cdot \omega \cdot \bar{\omega}
$$

and

$$
\# E(k)=(q-1)-(\pi-1)-(\bar{\pi}-1) .
$$

If $l$ is prime to $\omega$, then (3) yields $2 x=v_{l}(\# E(k))$ and (4) yields

$$
v_{l}(q-1) \geqslant \min \left\{x, v_{l}(\# E(k))\right\} \geqslant\left[\frac{v_{l}(\# E(k))}{2}\right] \text {. }
$$

This proves (2). If $l$ is not prime to $\omega$, then either $l$ is decomposed or is ramified in $O_{K}$. Suppose $(l)=\mathscr{L} \cdot \overline{\mathscr{L}}$ in $O_{K}$ with $\mathscr{L} \neq \overline{\mathscr{L}}$. Let, for example, $v_{\mathscr{L}}(\omega)>0$. Then $v_{\overline{\mathscr{L}}}(\omega)=0$ and $v_{\mathscr{L}}(\omega+\bar{\omega})=0$. Equation (3) yields $2 x<v_{l}(\# E(k))$ and Eq. (4) yields $x \geqslant \min \left\{v_{l}(\# E(k)), v_{l}(q-1)\right\}$, where equality holds if $v_{l}(\# E(k))$ and $v_{l}(q-1)$ are different. A detailed examination of the possible values of $v_{l}(\# E(k))$ and $v_{l}(q-1)$ shows that (2) holds. Suppose $(l)=\mathscr{L}^{2}$ in $O_{K}$. If $v_{\mathscr{L}}(\omega)>0$, then $v_{\mathscr{L}}(\omega)=1$. Equation (3) yields $2 x+1=v_{l}(\# E(k))$. Thus we get

$$
x=\frac{v_{l}(\# E(k))-1}{2}=\left[\frac{v_{l}(\# E(k))}{2}\right] \text {. }
$$

Equation (4) shows that $v_{l}(q-1) \geqslant\left[v_{l}(\# E(k)) / 2\right]$, which proves (2).

We can now give an answer to the first question and prove the following theorem.

THEOREM 3. Let $k$ be a finite field with $q=p^{n}$ elements. Let $h=\prod_{l} l^{h_{l}}$ be a possible order \#E(k) of an elliptic curve $E$ over $k$. Then all the possible groups $E(k)$ with $\# E(k)=h$ are the following:

$$
\mathbf{Z} / p^{h_{r}} \mathbf{Z} \times \prod_{l \neq p}\left(\mathbf{Z} / l^{a_{l}} \mathbf{Z} \times \mathbf{Z} / l^{h_{1}-a_{l}} \mathbf{Z}\right)
$$

with

(a) In case (b) of Theorem 1a: Each $a_{1}$ is equal to $h_{l} / 2$;

(b) In cases (a), (c), (d), (e) of Theorem 1a: $a_{l}$ is an arbitrary integer satisfying $0 \leqslant a_{l} \leqslant \min \left\{v_{l}(q-1),\left[h_{l} / 2\right]\right\}$.

Proof. (a) In case (b) of Theorem 1 a we get $\pi \in \mathbf{Z}$ and $h=(\pi-1)^{2}$. Furthermore, $\pi-1$ is divisible by $m$ in $\operatorname{End}(E)$ if and only if $\pi-1$ is divisible by $m$ in $\mathbf{Z}$. Hence Lemma 1 shows that $a_{l}=\min \left\{v_{l}(\pi-1),\left[h_{l} / 2\right]\right\}=h_{l} / 2$.

(b) Let $\{1, \eta\}$ be an integral basis of $O_{K}$. Then $\pi=a+b \eta$ with $a, b \in \mathbf{Z}$ and $b \neq 0$. This yields $\pi-1=a-1+b \eta$ with

$$
\min \left\{v_{l}(a-1), v_{l}(b)\right\}=\min \left\{v_{l}(q-1),\left[h_{l} / 2\right]\right\}
$$

by Lemma 2 . For each $l \neq p$ let $a$, be arbitrary with

$$
0 \leqslant a_{l} \leqslant \min \left\{v_{l}(q-1),\left[h_{1} / 2\right]\right\} .
$$

Consider the order $R$ in $O_{K}$ whose conductor is equal to $\prod_{l \neq p} l l^{\prime}(l) \cdots a_{l}$. There is an elliptic curve $E$ over $k$ with $R=\operatorname{End}(E)$ by Theorem 1b. The exact $l$-power that divides $\pi-1$ in $R$ is equal to $l^{a_{l}}$ for each $l \neq p$. Hence Lemma 1 shows that $E(k)$ is equal to $\mathbf{Z} / p^{h_{r}} \mathbf{Z} \times \prod_{l \neq p}\left(\mathbf{Z} / l^{a_{1}} \mathbf{Z} \times \mathbf{Z} / l^{h_{l}-a_{l}} \mathbf{Z}\right)$.

Department of Mathematics

University of Arizona

Tucson, Arizona 85721

FB9-Mathematik

Universität des Saarlandes

D-6600 Saarbrücken, West Germany 
1. M. Deuring, "Die Typen der Multiplikatorenringe elliptischer Funktionenkörper," Abh. Math. Sem. Hamburg, v. 14, 1941, pp. 197-272.

2. J. TATE, "Endomorphisms of abelian varieties over finite fields," Invent. Math., v. 2, 1966, pp. 134-144.

3. J. TATE, Classes d'Isogénie des Variétés Abéliennes sur un Corps Fini (d'après T. Honda), Séminaire Bourbaki, Exposé 352, Benjamin, New York, 1968/69.

4. W. WaterhouSE, "Abelian varieties over finite fields," Ann. Sci. École Norm. Sup. (4), v. 2, 1969, pp. $521-560$.

5. A. WeIL, Variétés Abéliennes et Courbes Algébriques, Hermann, Paris, 1948. 